\section{Handekzem: Was tun beim Rezidiv?}

\author{
Für Patienten mit therapierefraktärem chronischem Handekzem \\ bietet Alitretinoin seit zwei Jahren eine Behandlungsoption. Wie \\ effektiv ist diese Therapie bei erneuter Symptomverschlechterung?
}

\begin{abstract}
A litretinoin, ein Vitamin-A-Derivat, greift inhibierend in verschiedene Entzündungsprozesse ein. In der BACH-Studie (Benefit of Alitretinoin in Chronic Hand Ekzema) erwies sich die systemische Gabe dieser Substanz bei chronischem Handekzem, das auf eine lokale Glukokortikoidtherapie nicht bzw. ungenügend ansprach, als wirksame Therapie. Für diese Indikation ist Alitretinoin in mehreren europäischen Ländern bereits zugelassen.

In einer aktuellen Studie wurden 117 Patienten mit chronischem Handekzem aufgenommen, die in der BACH-Studie auf die Behandlung gut angesprochen hatten und von den Ärzten als „frei“ oder „fast frei“ von Ekzem beurteilt worden waren. Bei ihnen war es innerhalb von 24 Wochen zum Rezidiv gekommen - in dieser Zeit waren als Behandlung nur Emollienzien zugelassen. Die Studienteilnehmer erhielten randomisiert einmal täglich ent-
\end{abstract}

weder ihre frühere Studienmedikation oder Plazebo (30 mg Alitretinoin vs. Placebo, $10 \mathrm{mg}$ Alitretinoin vs. Placebo) für 12-24 Wochen. Als erneutes Ansprechen war die Einstufung als „frei“ oder „fast frei" von Läsionen definiert.

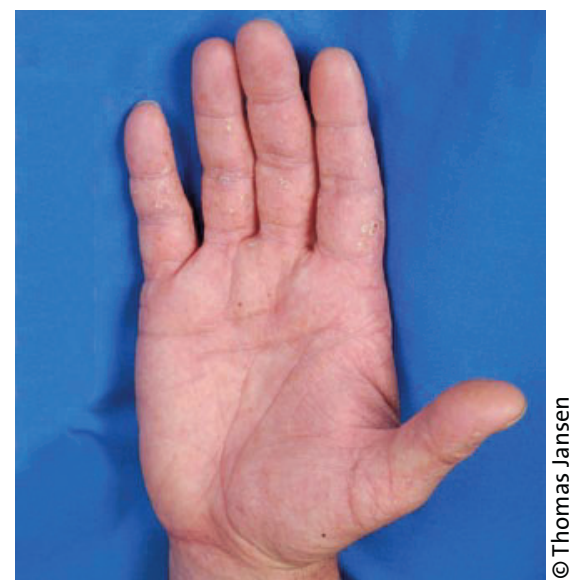

Konnte ein Handekzem durch Alitretinoin zur Abheilung gebracht werden, so ist bei einem Rezidiv ein erneuter Versuch durchaus gerechtfertigt.
In der 30-mg-Gruppe zeigte sich eine gegenüber Plazebo signifikant erhöhte Ansprechrate ( $80 \%$ vs. $8 \%$, p < $0,001)$. Unter der Gabe von $10 \mathrm{mg}$ Alitretinoin sprachen $48 \%$ der Studienteilnehmer an - gegenüber $10 \%$ unter Plazebo. Die Abnahme im modifizierten Total Lesion Symptom Score (mTLSS) lag nach 24 Wochen unter $30 \mathrm{mg}$ Alitretinoin bei $92 \%$, unter $10 \mathrm{mg}$ bei $71 \%$ und in der Plazebogruppe bei $43 \%$. An unerwünschten Wirkungen waren unter $30 \mathrm{mg}$ Alitretinoin Kopfschmerzen am häufigsten. Andere Nebenwirkungen traten nur geringfügig häufiger als unter Plazebo auf.

Fazit: Die Autoren schließen aus ihren Studienergebnissen, dass die Behandlung eines bisher therapierefraktären chronischen Handekzems mit Alitretinoin bei Respondern auch im Rezidivfall effektiv und gut verträglich ist. Demnach eignet sich die unterbrochene Behandlung mit diesem Wirkstoff auch zum Langzeitmanagement der Erkrankung.

Bissonnette R et al. Successful retreatment with alitretinoin in patients with relapsed chronic hand eczema.

Br J Dermatol 2010; 162: 420-6

\title{
Ekzem moduliert Krebsrisiko
}

\section{Ob Patienten mit atopischer Dermatitis ein erhöhtes oder geringeres Risiko für maligne Erkrankungen haben, wird kontrovers diskutiert. Eine differenzierte Aufschlüsselung nach Tumorentitäten sollte jetzt mehr Klarheit in die Zusammenhänge bringen.}

E ine atopische Dermatitis könnte einer Krebsentwicklung durch die permanente Immunstimulation vorbeugen - sie könnte durch diese Dauerstimulation aber auch zufällige proonkogene Mutationen fördern und damit das Krebsrisiko erhöhen. Bei differenzierter Betrachtung einzelner Tumorentitäten lässt sich dieser scheinbare Widerspruch auflösen. In Großbritannien wurde in einer retrospektiven Auswertung der Mitgliederdaten von "The Health Improvement Network" die Inzidenzrate des Erstauftretens verschiedener malig- ner Erkrankungen bei Personen mit und ohne atopische Dermatitis analysiert.

Eine Krebsdiagnose - ohne NichtMelanom-Hautkrebs (NMSC) - hatten 129.972 von insgesamt 4.518.131 Personen der Studienpopulation. Insgesamt ergab sich für Patienten mit atopischer Dermatitis ein erhöhtes Risiko für eine Krebserkrankung im Vergleich zu Hautgesunden. Die alters- und geschlechtsadjustierte Inzidenzrate (IRR) pro 10.000 Personenjahre betrug hier 1,49. Ein deutlich höheres Risiko bestand für die Ekzemerkrankten vor allem hinsichtlich eines Lymphoms (IRR 2,21). Die ebenfalls in dieser Studie gefundenen Risikoerhöhungen für Melanom (IRR 1,74) und NMSC (IRR 1,46) bedürfen einer Bestätigung in anderen Studien unter Berücksichtigung spezifischer Risikofaktoren zusätzlich zu Alter und Geschlecht.

Fazit: Patienten mit einer atopischen Dermatitis haben im Vergleich zu Hautgesunden ein erhöhtes Risiko für Lymphome und scheinbar auch für Melanome und Nicht-Melanom-Hautkrebs. Weitere Studien müssten nun klären, ob die Risikoerhöhung auf die atopische Dermatitis selbst oder möglicherweise auf deren Therapie zurückzuführen ist. $\quad b k$

Arana A et al. Incidence of cancer in the general population and in patients with or without atopic dermatitis in the U.K. Br J Dermatol 2010; 163: 1036-43 